\title{
A Fully Automated Cell Segmentation and Morphometric Parameter System for Quantifying Corneal Endothelial Cell Morphology
}

\author{
Shumoos Al-Fahdawi ${ }^{1}$, Rami Qahwaji ${ }^{1}$, Alaa S. Al-Waisy ${ }^{1}$, Stanley Ipson ${ }^{1}$, Maryam Ferdousi ${ }^{3}$, Rayaz A. \\ Malik $^{2,3}$, Arun Brahma ${ }^{4}$ \\ ${ }^{1}$ School of Electrical Engineering and Computer Science, University of Bradford, Bradford, UK. \\ ${ }^{2}$ Division of Medicine, Weill Cornell Medicine-Qatar, Doha, Qatar. \\ ${ }^{3}$ Institute of Cardiovascular Medicine, University of Manchester and the Manchester Royal Infirmary, Central Manchester Hospital Foundation Trust, \\ Manchester, U.K. \\ ${ }^{4}$ Manchester Royal Eye Hospital, Central Manchester University Hospitals NHS Foundation Trust, Manchester Academic Health Science Centre, Manchester, \\ $M 139 W L, U K$
}

\begin{abstract}
Background and Objective: Corneal endothelial cell abnormalities may be associated with a number of corneal and systemic diseases. Damage to the endothelial cells can significantly affect corneal transparency by altering hydration of the corneal stroma, which can lead to irreversible endothelial cell pathology requiring corneal transplantation. To date, quantitative analysis of endothelial cell abnormalities has been manually performed by ophthalmologists using time consuming and highly subjective semi-automatic tools, which require an operator interaction. We developed and applied a fully-automated and real-time system, termed the Corneal Endothelium Analysis System (CEAS) for the segmentation and computation of endothelial cells in images of the human cornea obtained by in vivo corneal confocal microscopy.

Methods: First, a Fast Fourier Transform (FFT) Band-pass filter is applied to reduce noise and enhance the image quality to make the cells more visible. Secondly, endothelial cell boundaries are detected using watershed transformations and Voronoi tessellations to accurately quantify the morphological parameters of the human corneal endothelial cells. The performance of the automated segmentation system was tested against manually traced ground-truth images based on a database consisting of 40 corneal confocal endothelial cell images in terms of segmentation accuracy and obtained clinical features. In addition, the robustness and efficiency of the proposed CEAS system were compared with manually obtained cell densities using a separate database of 40 images from controls $(n=11)$, obese subjects $(n=16)$ and patients with diabetes $(n=13)$.

Results: The Pearson correlation coefficient between automated and manual endothelial cell densities is 0.9 ( $\mathrm{p}<0.0001$ ) and a Bland-Altman plot shows that $95 \%$ of the data are between the 2SD agreement lines.

Conclusions: We demonstrate the effectiveness and robustness of the CEAS system, and the possibility of utilizing it in a real world clinical setting to enable rapid diagnosis and for patient follow-up, with an execution time of only 6 seconds per image.
\end{abstract}

Keywords: Corneal Endothelial Cells, Automatic Cell Segmentation, Corneal Confocal Microscopy, Fast Fourier Transform, Watershed Transformation, Voronoi Tessellation.

\section{Introduction}

In vivo Corneal Confocal Microscopy (CCM) is a fast noninvasive clinical technique for acquiring images and quantifying morphological changes in the cornea to provide insights into a range of endothelial pathologies and infections [1][2]. As shown in Fig.1, the cornea is the anterior transparent part of the eye, which transfers and focuses light onto the retina. It is composed of five different layers, covering crucial internal structures of the human eye, including the iris, pupil, lens, and anterior chamber [3][4]. Corneal transparency is primarily dependent on corneal stromal hydration, which is maintained by an active transport mechanism in the corneal endothelium [5]. The corneal endothelium is a connected single-layer of hexagonal uniformly sized cells on the posterior surface of the human cornea [6]. Several factors can damage this regular tessellation and cause cell loss, including aging, intraocular surgery, inflammation or other ocular or systemic pathologies [7]. Damage to the endothelial cells can lead to altered hydration of the corneal stroma and visual loss, which may be associated with irreversible endothelial cell pathology requiring corneal transplantation (Keratoplasty) [8]. The corneal endothelial loss is compensated by an enlargement and migration of neighbouring cells due to a lack of regenerative capacity of the corneal endothelium. This results in a decrease in cell density, increase in the variation of the cell surface area and deformation of the hexagonal pattern of endothelial cells, which can cause disruption of endothelial layer function as a fluid barrier [9].
In-vitro quantitative analysis of the corneal endothelium is currently undertaken at eye hospitals to assess the functional capacity of the corneal endothelium, and hence the quality of the donor cornea prior to transplantation [10]. A minimum Endothelial Cell Density (ECD) of 400 to $600\left(\right.$ cells $/ \mathrm{mm}^{2}$ ) is an indicator of corneal endothelial health and most donor corneas should have an ECD of at least $2000\left(\right.$ cells $\left./ \mathrm{mm}^{2}\right)$ to be authorized for Keratoplasty [7][11]. The corneal endothelium should also ideally have $100 \%$ Hexagonality, with $60 \%$ being accepted as an indicator of a healthy corneal endothelium [9]. The most commonly used features to quantify endothelial cell health are: Endothelial Cell Density (ECD) $\left(\right.$ cell $\left./ \mathrm{mm}^{2}\right)$, polymegathism (Coefficient of Variation in cell size), pleomorphism (Percentage of Hexagonality Coefficient), Mean Cell Area (MCA) $\left(\mu \mathrm{m}^{2}\right)$ and Mean Cell Perimeter (MCP). However, these features are not frequently used in the clinical setting due to the considerable errors of cell boundary detection [12]. Recently even healthy control subjects have been shown to have significant differences in ECD and pleomorphism in central and peripheral areas of the cornea [1]. To date, the quantitative analysis of the corneal endothelium has been manually performed by visual inspection of images by ophthalmologists. ECD is derived by experts counting all the endothelial cells inside a selected Region of Interest (ROI) aided using a digital image tool that allows them to place a mark on each endothelial cell [10]. This manual procedure is tedious, time-consuming, highly subjective, and error prone, and does not allow the geometric analysis of endothelial cell shape [5]. This limits the quantification of the additional morphometric features to clinical research and does 
not allow adoption for routine clinical use. However, the additional morphometric features can be easily measured if the endothelial cell boundaries are correctly identified [6]. An objective and fully-automated segmentation and quantification system enabling rapid quantitative analysis of the corneal endothelium would facilitate translation to the clinical setting. Whilst several prototype systems have been proposed to automatically detect endothelial cell boundaries, the quality of the captured images (e.g., images are often blurred and noisy) can result in significant issues in the detection of cell boundaries requiring operator interaction to guide the detection process and hence reducing the speed of analysis, more details are provided in Section 2.

In this paper, a totally automatic, robust and real-time system is proposed, termed the Corneal Endothelium Analysis System (CEAS) for the segmentation and computation of the different morphological parameters of endothelial cells in the human cornea obtained by in vivo corneal confocal microscopy. First, an FFT-Band-pass filter is applied to reduce noise and enhance the image quality to make the cells more visible. Secondly, a watershed transform and a Voronoi tessellation are applied to detect all the endothelial cells in the image, which facilitates the robust and accurate extraction of the endothelial cell contour. This paper is organized as follows: Related endothelial research work is discussed in Section 2. The description of the materials used and the proposed endothelium segmentation and quantification system are explained in Section 3. The experimental results are presented in Section 4. Finally, conclusions and future research directions are presented in Section 5.

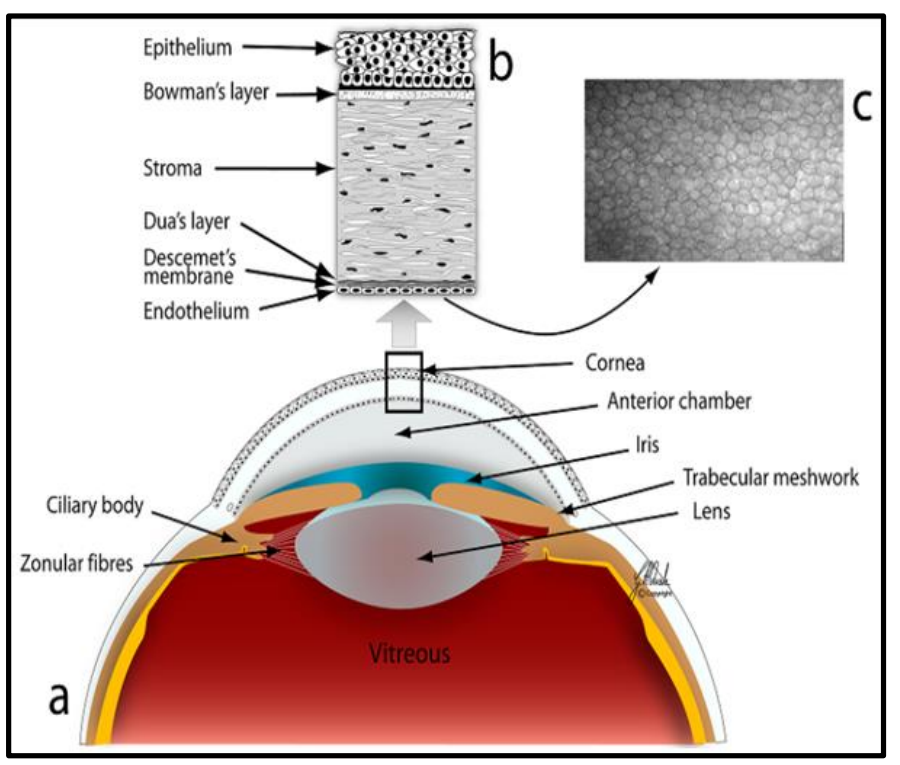

Figure 1. The anatomy of the human eye and the cornea (a) Section of the frontal part of the human eye, (b) Six layers from the anterior to the posterior cornea, (c) In vivo corneal confocal microscopy image of the corneal endothelium layer [8]

\section{Discussion}

Several researchers have shown an increased interest in the field of automatic segmentation of corneal endothelial cell images. Foracchia and Ruggeri [13] proposed a corneal endothelial cell segmentation approach based on a neural network architecture whose weighted parameters (numerical filters) were specifically prepared for a border detection problem and obtained from the Boundary Contour System (BCS). In this study, the "expert correction" approach was proposed to recover missing boundaries and tentative splitting or merging of cell bodies. However, this algorithm used endothelial images collected from 3 different ophthalmic instruments with no information given about these instruments. In 2005, Ruggeri et al. [10] presented a fully automated estimation algorithm for endothelial cell density based on extracting the spatial frequencies included in digital endothelial images using a 2DDiscrete Fourier Transform (DFT) approach. The frequency information obtained from a circular band in the 2D-DFT of the endothelial images contains information related to the endothelial cell density. The performance was evaluated on 100 corneal endothelial images obtained by following the same procedures usually employed at the Berlin Cornea Bank. The endothelial images of these corneas were acquired using an inverse phase contrast microscope. The mean difference between automated and manual densities was 14 (cells $\left./ \mathrm{mm}^{2}\right)$, with a standard deviation of 119 (cells $/ \mathrm{mm}^{2}$ ) and the running time was (1-2) seconds per image. An approach to derive the density of endothelial cells without determining the cell boundaries was also proposed in [14] and assumes an approximately regular tessellation of hexagonal shapes. The approach calculates the inverse transpose of a matrix (aka basis) producing this cellular lattice and is used to estimate the cell density. Due to the different sizes and spatial orientations of endothelial cells throughout the image, the basis matrix could differ significantly from one region to another and a local estimation is performed to reduce the effects of this variability. The performance of this approach was evaluated on a set of 21 corneal endothelial images captured by an inverse phase-contrast microscope in the Berlin corneal bank. The mean difference between the manual and automated endothelial cell densities was $-0.1 \% \quad(6.5 \%$ for absolute differences). Foracchia and Ruggeri [5] have presented a new automatic detection and analysis approach based on a set of single cell boundary models, which statistically describe individual endothelial cells in terms of shape a priori information and a-posteriori image representation. Each cell is individually determined (by Maximum Posteriori estimation) in an image given a starting point and a Simulated Annealing (SA) as an optimization algorithm. While a cell field is estimated, further information is introduced and the overall model identification is improved by using the interaction between cell models. The results show an improvement in the detection of cell contours of specular microscope images. Hiroyasu et al. [15] proposed a corneal endothelial cell segmentation system based on constructing a tree-structural image-processing filter, which can be applied to images of regions with different statistics. This system produces two types of nodes (e.g., one type represents well-known image-processing filters and the second represents conditional branches), their combination is optimized using genetic programming (GP). However, experiments were undertaken on only two corneal endothelial images captured using a phase-contrast microscope.

Scarpa and Ruggeri [6] proposed a segmentation system identifying endothelial cell boundaries based on a genetic algorithm technique. The operation of the genetic algorithm is mainly dependent on combining information about the model regularity of endothelial cell appearance with the intensity of the actual pixels in the corneal image. 15 corneal endothelial images captured with a specular endothelial microscope were compared with ground truth acquired from manually drawn endothelial cell boundaries. The average difference between the manual and automated approach was $4 \%$, and the maximum difference was lower than 7\%. Poletti and Ruggeri [16] have also presented an analysis method based on a supervised classification system for endothelial cell segmentation. This method was used to extract the cell boundary polygon in terms of its three elements: vertices, sides and body, employing a multi-scale approach with 2D matched filters. In particular, three kernels were prepared to 
extract the three endothelial cell components' signatures. These components' signatures were used as features to train a Support Vector Machine (SVM) classifier, to provide the final endothelial cell segmentation. The performance of the suggested method was evaluated on a set of 20 images acquired by in-vivo specular microscopy and the running time was 5 to 10 seconds per image. Two approaches to analyze and quantify corneal endothelial cells captured by in vivo white light slit-scanning confocal microscopy were presented by Selig et al. [4]. The first approach depends on a spatial frequency spectrum analysis approach to evaluate the ECD. In the second approach, endothelial cells are automatically segmented by employing a stochastic watershed approach after randomly placing seeds over the whole image. Due to noise in the input image, and hence over segmentation, a smoothing filter with a Gaussian kernel and H-minima transform is applied before applying the stochastic watershed approach to estimate endothelial cell density, polymegathism and pleomorphism. However, in some cases an operator interaction is required to correct the final segmented results, which can take approximately 30 seconds and altogether 4 minutes are required to estimate the cell density, limiting real-time application in the clinic. The performance of the two algorithms was evaluated on a set of 52 corneal endothelial images captured from 23 patients using a white-light slit-scanning confocal microscope and compared with the NAVIS software. Sharif et al. [3] developed a hybrid model for analyzing confocal endothelial images based on a combination of Active Contour version of the Snake (S) model and a Particle Swarm Optimization (PSO) approach (S-PSO). Firstly, a pre-processing procedure was employed using DFT combined with a Band-pass Butterworth filter to enhance the quality and reduce the noise level of the input image. Then, boundaries of the corneal endothelial cells were traced using the (S-PSO) approach. Results from 11 abnormal confocal endothelial images were compared with manual and two other approaches based on morphological operations. The mean differences between manual and automated cell densities were $5 \%, 7 \%$ and $13 \%$, respectively. Recently, several studies have proposed supervised approaches to accurately detect the endothelial cell contours in images captured using specular microscopy. For instance, Katafuchi and Yoshimura [17] proposed a new segmentation algorithm based on Convolutional Neural Network (CNN) to detect the cell contours regardless to the scale of cells. Fabijanska [18] developed an efficient algorithm to address the problem of corneal endothelium image segmentation using Feed-Forward Neural Network (F-FNN), trained to recognize pixels whether they belong to the cell borders or not. However, the main issue of using the supervised segmentation approaches is the time required for training the neural network in order to be able to accurately detect the cell contours. Finally, Gavet and Pinoli [19] presented a supervised segmentation evaluation methodology to compare between three endothelial cell segmentation methods, namely: Vincent and Masters' method, Angulo and Matou's method and Gavet and Pinoli's method. In this study, a database consisting of 30 greytone images of the human corneal endothelium captured with a specular microscope was employed to evaluate these segmentation methods. Habrat et al. [20] proposed an algorithm for the detection of corneal endothelium cells in images obtained with confocal microscopy, termed as $\mathrm{KH}$ algorithm. The $\mathrm{KH}$ algorithm starts by reducing the effects of the noise and nonuniform illumination in endothelial images using a binary filter of size $(5 \times 5)$ pixels. This is followed by applying the binarization process using four morphological operators of size $(9 \times 9)$ pixels; two of them were rotated by $45^{\circ}$ and the remaining two by $90^{\circ}$. As a result, four different binary images were obtained by convolving the original image with these four filters. Finally, these four images were fused together to produce the final output after removing all the objects smaller than 40 pixels, which probably correspond to cell nuclei.

This review on corneal endothelial cells segmentation and quantification systems has highlighted a number of limitations which need to be addressed. Firstly, most previous studies have used images acquired using a specular microscope which provides high contrast between the different endothelial tissues and creates good, high contrast, images with trivial light dispersal. Despite increasing use of in vivo corneal confocal microscopy for both clinical and research purposes, little research has been undertaken to develop a fast and fully-automated segmentation algorithm for quantifying corneal endothelial images acquired with in vivo corneal confocal microscopy. Secondly, most studies have utilized very small sets of corneal endothelial images which are insufficient to reveal the real-world performance of the proposed approaches. Finally, some systems require an operator interaction to revise the incorrect results presented in the final segmented image, including missing cell boundaries and merging or splitting of cell bodies. A rapid objective user-independent fully-automatic endothelial cell segmentation and quantification system is required to overcome these issues.

\section{The Proposed Methodology}

The proposed CEAS system is a fully-automated system which requires no user intervention to accurately detect the cell contours. Unlike, other supervised segmentation approaches [17][18], which require a long time for training the neural network to detect the cell contours, no training procedure is required using the proposed CEAS system. It also enables the quantification of the additional morphometric features (e.g. polymegathism, pleomorphism, etc.), which is a limitation in many of the built-in tools. For example, the built-in software included in the HRT Rostock Cornea Module (Heidelberg Engineering $\mathrm{GmbH}$; Heidelberg; Germany) can only measure the cell density. As depicted in Fig.2, the proposed CEAS system consists of two essential stages: a cell segmentation stage and a morphometric parameter quantification stage. The former stage can be further divided into two steps: a preprocessing step to enhance the image quality and a cell contour detection step to accurately detect the cell boundaries. In the latter stage, a number of useful clinical parameters are calculated, including: Mean Cell Density (ECD), Polymegathism, Pleomorphism, Mean Cell Area (MCA) and Mean Cell Perimeter (MCP). These additional morphologic parameters may play a significant role in the early diagnosis of corneal pathology and in determining the health status of corneas for transplantation.

\subsection{Materials}

In this paper, a total of 80 images of corneal endothelial cells were acquired using a laser CCM (Heidelberg Retinal Tomograph III Rostock Cornea Module HRT III RCM; Heidelberg Engineering GmbH; Heidelberg; Germany) according to an established protocol [21]. The images were taken from the central cornea using the section mode and saved in BMP format with 8 -bit grey levels and size $(384 \times 384)$ pixels $(400 \times 400 \mu \mathrm{m})$, corresponding to a square pixel of size $1.0417 \mu \mathrm{m}$. Examples of original, unprocessed images of the endothelial cell layer are shown in Fig.3. It is important to note that the images used in this paper were extremely challenging with the very low quality compared to those in the literature. These images were divided into two databases, each containing 40 images. 


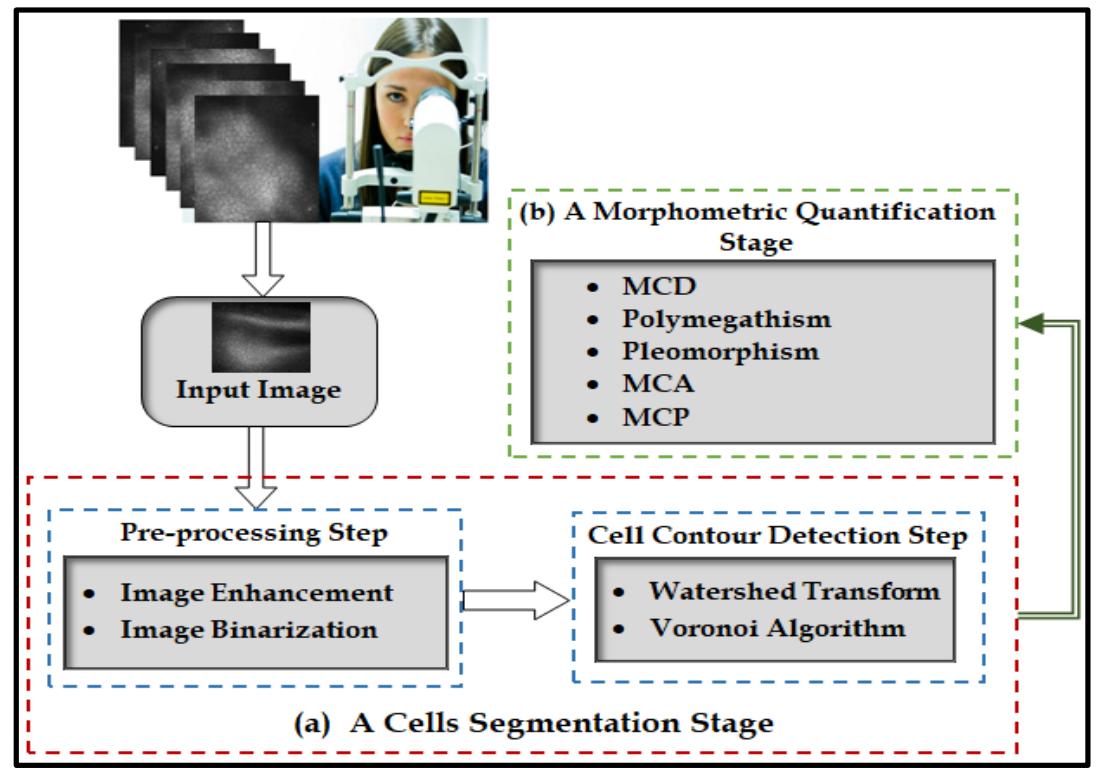

Figure 2. An illustration of the proposed CEAS system: (a) a cell segmentation stage and (b) a morphometric quantification stage.

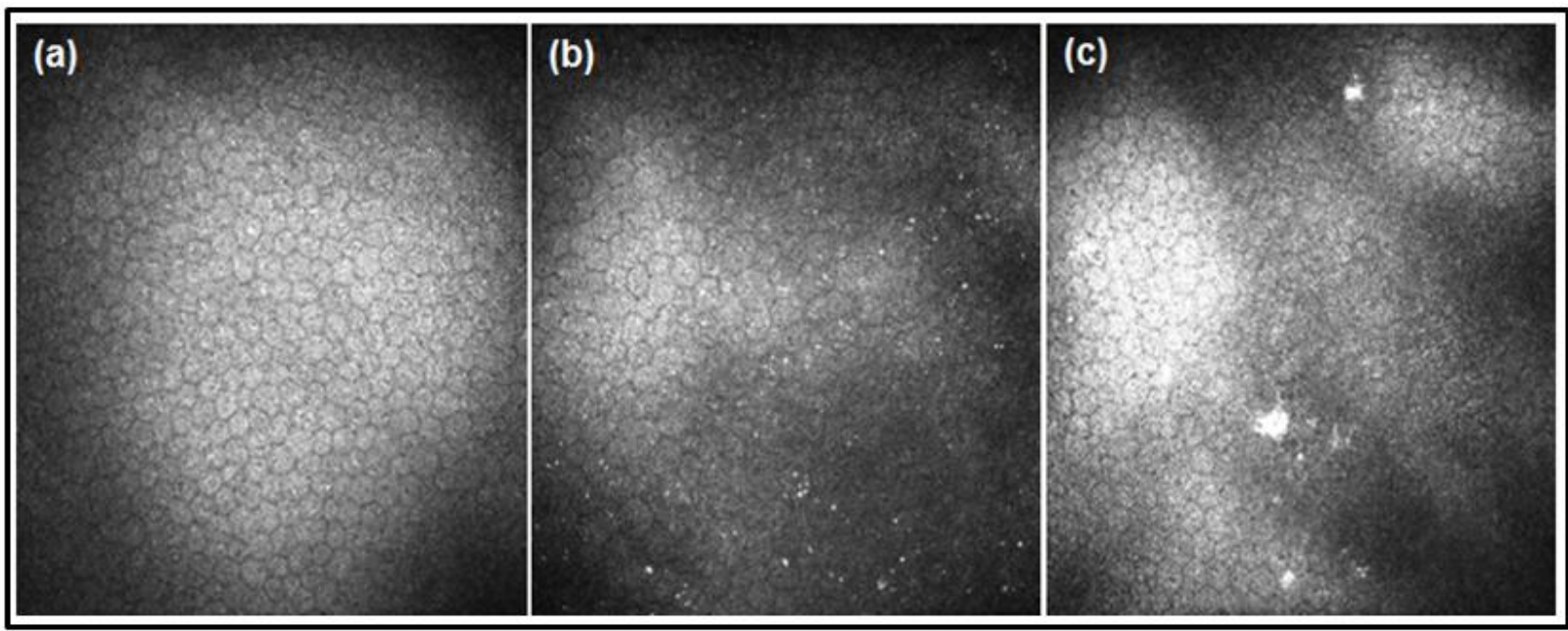

Figure 3. Examples of original corneal endothelial cell images from: (a) Control subject, (b) Morbidly obese patient, and (c) Diabetic patient showing a lower ECD and alteration in the size and shape of cells.

\subsection{Pre-processing Step}

The images acquired using CCM usually suffer from different types of artefact (e.g., blurring, noise, specular reflections, low contrast and non-uniform illumination) which makes the accurate detection of the cell contour a challenging task (Fig.3). The main reasons for the poor quality images are related to a number of factors in the acquisition process [22][2] including: (i) saccadic eye movement during image acquisition resulting in blurred images, (ii) differences in the pressure applied between the CCM Tomocap and cornea, (iii) the spherical shape of the cornea leads to non-regular distribution of the lighting in different corneal areas. The pre-processing stage aims to address these problems. An FFT based band-pass filter is applied for noise reduction to enhance the image quality and make the cell borders more visible, especially when there is a significant difference in pixel intensity between the inner cell bodies and intercellular space [23][24]. Firstly, an input image is transformed into a 2D representation of FFT's frequencies, and then a simple Band-pass filter is applied to suppress the frequency coefficients below and above a low and high threshold, respectively. In this work, Bandpass high and low frequency cut-offs are set to be 20 pixels and 3 pixels, respectively. Thus, the noise and slow variations in illumination are eliminated [25][26]. This is followed by applying the Inverse Fast Fourier Transform (IFFT) to transfer the image back into the spatial domain [2]. In this paper, the FFT-Band-pass filter is applied six times, and each time we observe that the cell boundaries are significantly enhanced, especially in the dark regions at the corner of the images. The output of this step is shown in Fig.4 (b). Next, an image binarization process is applied using a mean value threshold, which is automatically determined using a grey level histogram of the input image. In this process, all pixels in an endothelial image having intensity values less than the pre-defined threshold are set to 0 (black pixels), while the rest are set to 1 (white pixels). As shown in Fig.4 (c), the binarized image consists of circular cell markers which sometimes are linked with each other in a rosary manner. In this paper, these linked markers are separated by applying the watershed approach on top of the Euclidian distance map which is computed from the binarized image, explained in the next sub-section.

\subsection{Cell Contour Detection Step}

In this stage, an efficient segmentation algorithm based on watershed transformation and Voronoi Tessellation approach is employed to efficiently and automatically detect the endothelial cell boundaries. The watershed transformation approach is applied to the pre-processed image after calculating its Euclidean distance map to automatically separate merged markers obtained from the pre-processed image. 


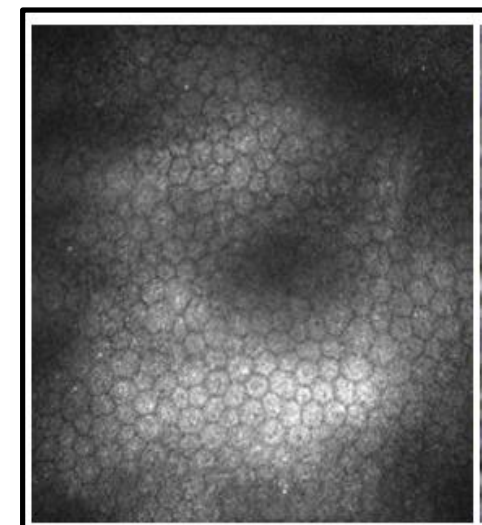

(a)

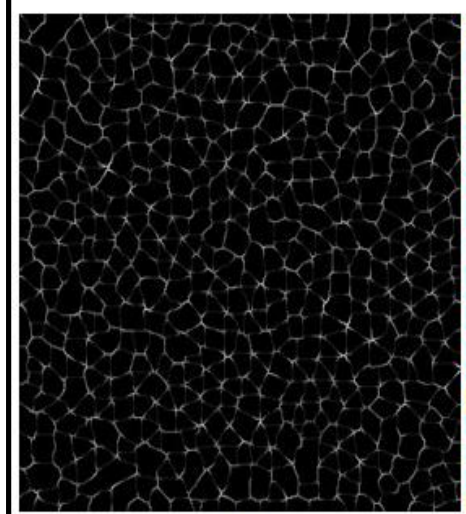

(e)

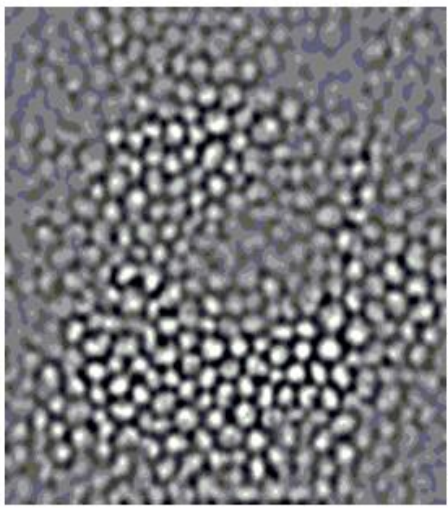

(b)

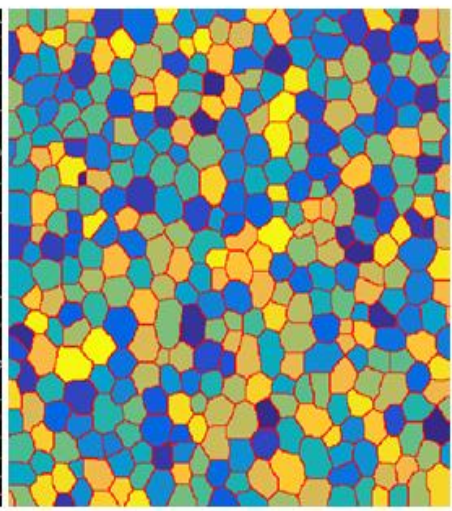

(f)

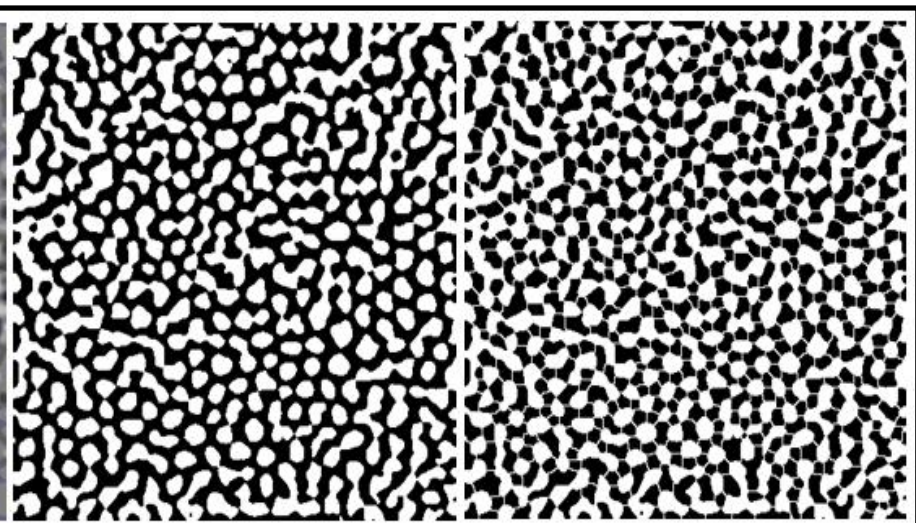

(c)

(d)

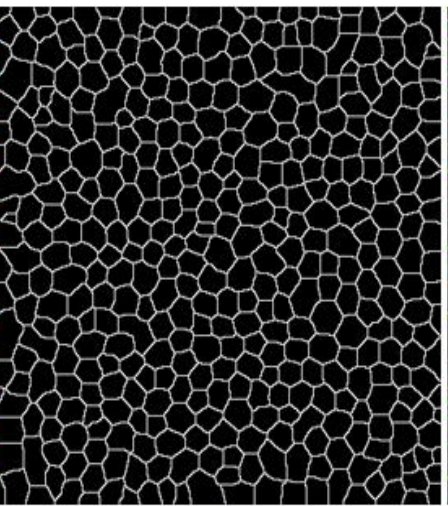

(g)

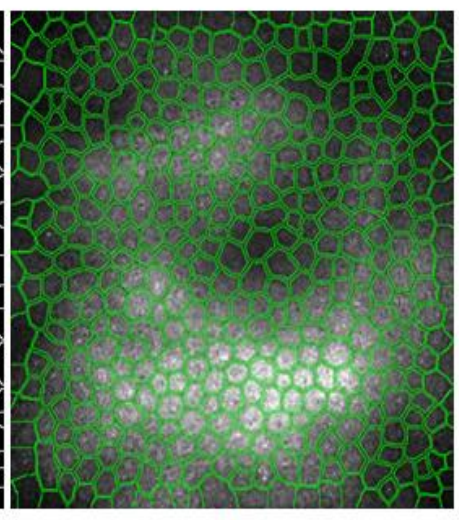

(h)

Figure 4. Corneal cell segmentation system outputs: (a) Original corneal image, (b) Applying FFT-Band-pass filtering, (c) Binarized image, (d) Applying watershed approach, (e) Applying Voronoi tessellation, (f) Labeling of endothelial cells (g) Final endothelial cells segmentation result, and (h) Automatically traced endothelial cells boundaries.

The Euclidean distance map has each original black pixel replaced by the value of its distance to the nearest edge pixel to generate a clearer grey level image. Therefore, the centre of each cell will be represented by the smallest value, as it represents the farthest point from the cell borders. This generated Euclidean distance map is then reinterpreted as a topographic map with its pixel values representing altitude which can be easily identified, with mountains, valleys and water catchment regions using the watershed approach, as water flows downhill in any direction from mountains (peaks and ridges) to valleys (lowest points) [27][28][29]. Here, the main aim of the watershed approach is to find the frontiers between the water catchment regions, and then the linked rosary markers in the binarized image are separated using these frontiers (Fig.4 d). In this paper, the watershed approach works best with smooth convex objects with less overlapping between them [27]. The Voronoi Tessellation approach is the second step of the proposed segmentation algorithm, which is applied to the output of the watershed approach in order to produce the final polygonal borders map using the coordinates of the cell centres as an input. Several studies have demonstrated the efficiency of the Voronoi Tessellation approach for morphometric cell analysis including the corneal endothelium [30][31]. Suppose that we have an image with a set of circular markers $\boldsymbol{M}=\left\{\boldsymbol{m}_{1}, \ldots, \boldsymbol{m}_{\boldsymbol{n}}\right\}$, a Voronoi Tessellation approach divides this image into $\boldsymbol{n}$ cells, one for each circular marker in $\boldsymbol{M}$ where each point $\boldsymbol{p}$ lies in the cell corresponding to a circular marker $m_{i}$ if $\operatorname{dist}\left(m_{i}, p\right)<\operatorname{dist}\left(m_{j}, p\right)$ for $\boldsymbol{i}$ distinct $^{1}$ from $\boldsymbol{j}$. The borders of endothelial cells are found by drawing lines of equidistant points between each two nearest circular markers' centres. In other words, these polygons produced from drawing lines around every centre marker represent the borders of endothelial cells. In the image produced, the pixel value inside each endothelial cell is set to zero, while the pixel values on the borders of the cells are equal to the distance between the two nearest marked centres (Fig.4 e-f).

\subsection{Morphometric Parameters Quantification Stage}

At this stage, a number of clinically useful features are extracted from the segmented endothelial cell images in an automated and objective manner to accurately describe the health of the corneal endothelial cells based on quantifying MCD $\left(\right.$ cell $\left./ \mathrm{mm}^{2}\right)$, polymegathism $(\%)$, pleomorphism $(\%), \operatorname{MCA}\left(\mu \mathrm{m}^{2}\right)$ and MCP $(\mu \mathrm{m})$. These extracted morphological features obtained with the proposed CEAS system are reported as a readable text file (Fig.5). Due to the poor quality of the captured images (e.g., some regions are of high reflectivity or they are extremely dark and blurred), accurate cell segmentation and estimation of the morphological features in these regions is very challenging (Fig.6 a). To address this issue and make the analysis more applicable clinically, the proposed system allows an ophthalmologist to choose and crop the clearest ROI in the segmented image. The morphological features are then calculated automatically only for the cropped region, by including cells that intersect only with two adjacent borders of the frame, and

\footnotetext{
${ }^{1}$ The distance between points is calculated using the Euclidean distance.
} 


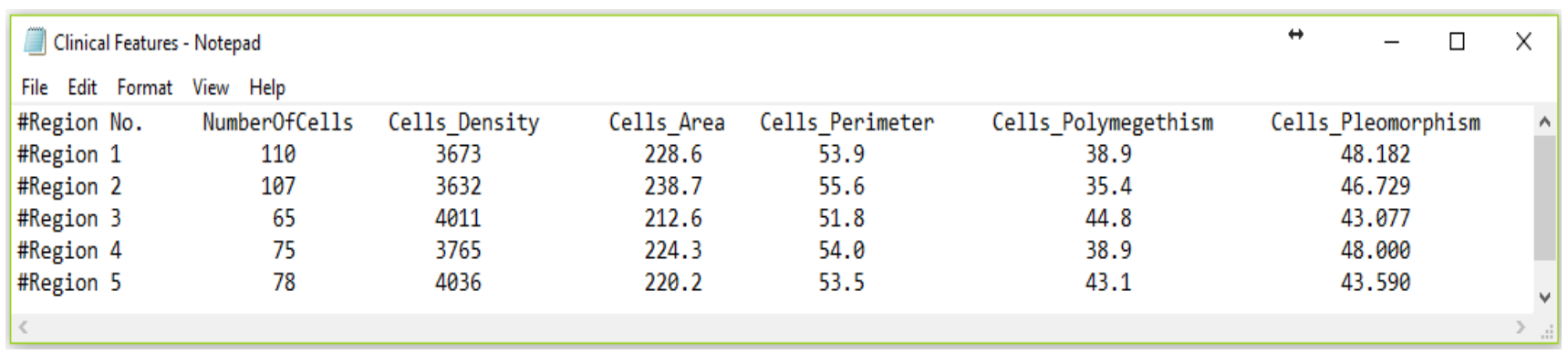

Figure 5. A readable text file format showing morphological features of the corneal endothelium.

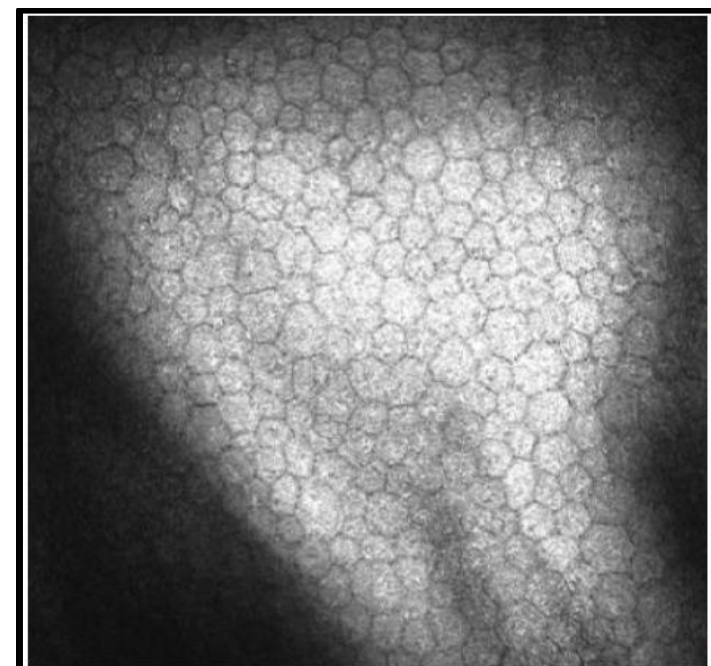

(a)

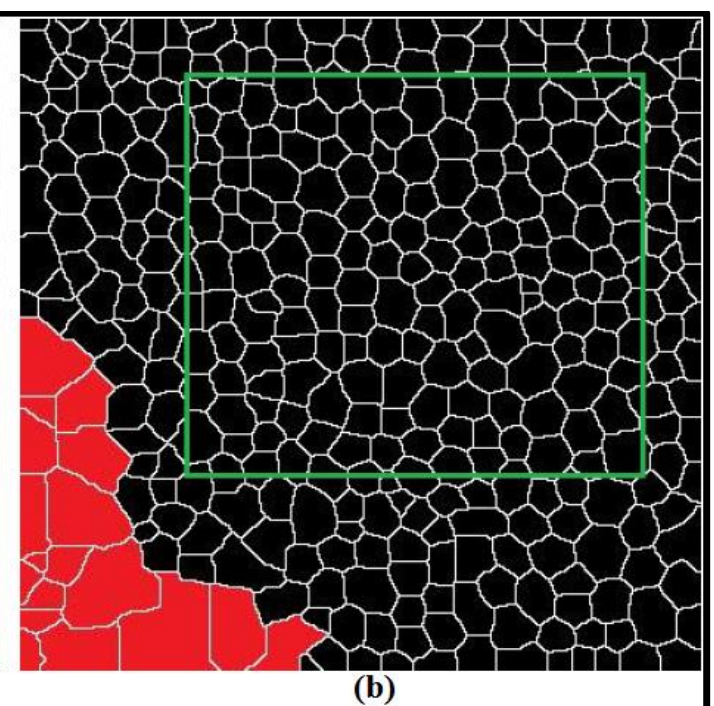

(b)

Figure 6. (a) Original corneal endothelial image and (b) Endothelial cell segmentation results with red color indicating the cells that have been ignored.

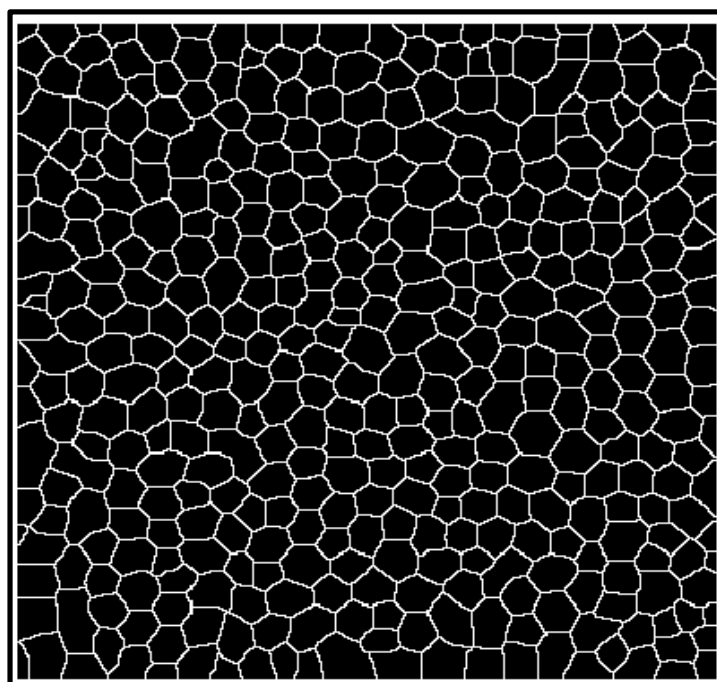

(a)

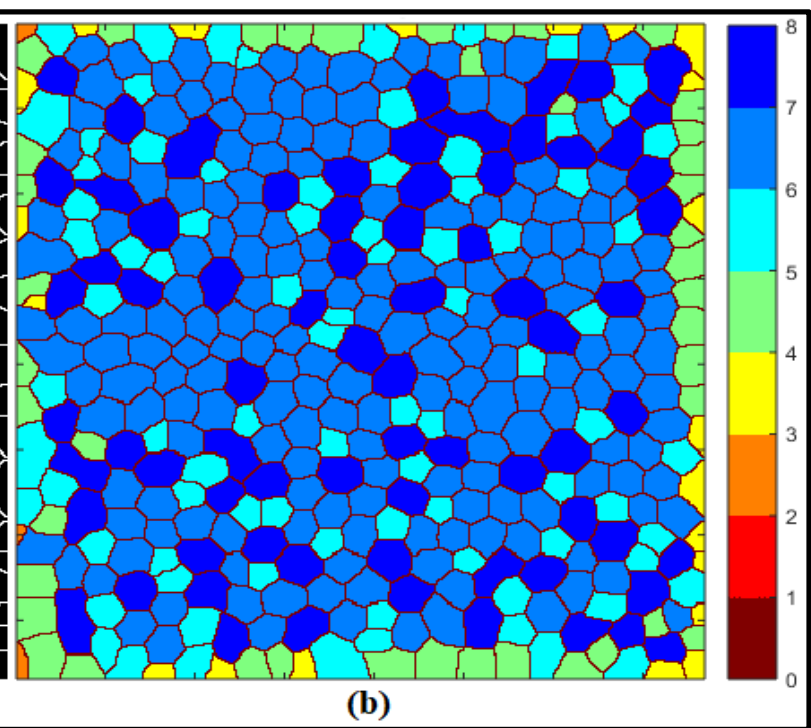

(b)

Figure 7. (a) Final endothelial cells segmentation result and (b) Using the color of a cell to indicate its number of neighbors using the color code given on the right. The most common color is orange corresponding to six neighbors.

excluding those intersecting with other borders. However, if the whole image is used, all the outermost cells are excluded from the statistical calculation to avoid any inaccurately segmented cells on the edge of the input image.

- Mean Cell Density (MCD) is calculated as the number of endothelial cells $\left(\boldsymbol{C}_{\text {number }}\right)$ in the cropped ROI (or whole image) divided by the total size $(\boldsymbol{A})$ of the cropped ROI (or whole image), as follows:

$$
M C D=\frac{C_{n u m b e r}}{A}
$$

- Polymegathism (Coefficient of Variation (CV)) is used to describe the variation in the area of the endothelial cells. An increase in the standard deviation $(\boldsymbol{S D})$ of the MCA leads to an inaccurate estimation for the MCD. Hence, an increase in polymegathism leads to a decrease in the accuracy of the estimated MCA [9]. Polymegathism is calculated as follows:

$$
\text { Polymegathism }=\frac{S D_{\text {cell area }}}{M C A} * 100
$$

Here, $\boldsymbol{S} \boldsymbol{D}_{\text {cell area }}$ is the standard deviation of cell area divided by the MCA. 
- Pleomorphism (Hexagonality Coefficient (HC)) is calculated as the number of cells with an approximately hexagonal shape (Six-sided) $\left(\boldsymbol{C}_{\text {hexagonal }}\right)$ divided by the total number of cells in the cropped ROI (or whole image) $\left(\boldsymbol{C}_{\text {image }}\right)$, as follows:

$$
\text { Pleomorphism }=\frac{C_{\text {hexagonal }}}{C_{\text {image }}} * 100
$$

The endothelial cells are shown in Fig.7 (a) in different colors. In Fig.7 (b) they are shown color coded with all cells with the same number of neighbors filled with the same color. Cells with six sides (roughly hexagonal in shape) are shown in sky blue color.

\section{Experimental Results}

A total of 80 images of corneal endothelial cells were acquired using a laser in vivo CCM to assess the performance of the proposed CEAS system. These images were divided into two databases, named Database_1 and Database_2, each one consisting of 40 images. However, due to the lack of availability of a manual dataset containing manual measurements for all the morphologic parameters obtained from these images, a manual version from each database was obtained by a cornea imaging expert from the University of Manchester using two different programs. Initially, the performance of the proposed CEAS system was evaluated on Database_1, where an open-source GNU Image Manipulation Program (GIMP) ${ }^{2}$ was used to manually trace cell contours and create a binary image from selected ROIs with a manual estimation of the morphometric parameters (Fig.8). Using Database_1, two experiments were conducted to assess the accuracy of the proposed CEAS system. In the first experiment, the performance of the proposed segmentation algorithm was evaluated against the ground-truth reference images (binary images) generated using GIMP software (Fig. 8 c). The evaluation procedure is based on the computation of the seven quantitative performance measures: Probabilistic Rand Index (PRI) [32], Structural SIMilarity (SSIM) Index [33], Gradient Magnitude Similarity Deviation (GMSD) [34], Variation of Information (VoI) [35], Mean Square Error (MSE), Normalized Absolute Error (NAE) [36] and Global Consistency Error (GCE) [37]. These full-reference quantitative metrics are widely employed in the literature for assessing the efficiency and accuracy of segmentation systems and are defined as follows:

1. The Probabilistic Rand Index (PRI) is a similarity function that counts the fraction of pairs of pixels whose labels are consistent between the segmented and the ground-truth images, through averaging across multiples of ground-truth images to account for scale-variation in human perception. The PRI value ranges between $\mathbf{0}$ and $\mathbf{1}$, and a higher value points to a better similarity.

2. The Structural SIMilarity (SSIM) Index is a full-reference metric for measuring the similarity between two images by taking the product of three types of similarities: Luminance Similarity (LS), Contrast Similarity (CS) and Structural Similarity (SS). Suppose that $\boldsymbol{X}$ and $\boldsymbol{Y}$ are the segmented images and the ground-truth image respectively, the overall quality measure of the entire image is obtained by calculating the mean of the SSIM index (MSSIM) as follows:

$$
\operatorname{MSSIM}(X, Y)=\frac{1}{M} \sum_{i=1}^{M} \operatorname{SSIM}\left(x_{i}, y_{i}\right)
$$

Here, $\boldsymbol{x}_{\boldsymbol{i}}$ and $\boldsymbol{y}_{\boldsymbol{i}}$ are the image contents within the $\boldsymbol{i}$-th local window, $\boldsymbol{M}$ is the number of local windows in the image and the MSSIM value ranges between $\mathbf{0}$ and $\mathbf{1}$, the higher value pointing to greater similarity.

3. The Gradient Magnitude Similarity Deviation (GMSD) is an image quality assessment method which computes the Local Quality Map (LQM) by locally comparing the gradient magnitude maps of segmented image $\boldsymbol{X}$ and ground-truth image $\boldsymbol{Y}$. This is followed by applying the standard deviation of LQM as the pooling strategy to produce the final quality score, as follows:

$$
G M S D=\sqrt{\frac{1}{N} \sum_{i=1}^{N}(G M S(i)-G M S M(i))^{2}}
$$

where, $N$ is the total number of pixels in the image, GMSM represents the mean of the GMS map, which is computed in a pixel-wise manner using the gradient magnitude images $\boldsymbol{m}_{\boldsymbol{x}}$ and $\boldsymbol{m}_{\boldsymbol{y}}$.

4. The Variation of Information (VoI) is a non-negative metric that measures the distance between segmentations (e.g., automatic and manual) in terms of the information loss and gain between them. The VoI metric mainly depends on entropy and mutual information to compute the distance between two segmentations. The VoI between segmented image $\boldsymbol{X}$ and the ground-truth image $\boldsymbol{Y}$ is computed as follows:

$$
\operatorname{VoI}(X, Y)=H(S)+H(Y)-2 I(X, Y)
$$

Here, $\boldsymbol{H}$ and $\boldsymbol{I}$ represent the entropy and the mutual information, respectively and they are computed as in [38]. The VoI value ranges between $\mathbf{0}$ and $\infty$, and a lower value indicates greater similarity.

5. The Mean Square Error (MSE) is one of the most widely used image quality measurement metrics, defined as the sum over all squared value pixel differences divided by the size of the image, where a lower value of MSE indicates a higher similarity. The MSE between the segmented image $\boldsymbol{X}$ and the ground-truth image $\boldsymbol{Y}$ of equal size $(\boldsymbol{M} \times \boldsymbol{N})$ pixel is defined as follows:

$$
M S E=\frac{I}{M N} \sum_{m=1}^{M} \sum_{n=1}^{N}(X(m, n)-Y(m, n))^{2}
$$

6. The Normalized Absolute Error (NAE) between the segmented image $\boldsymbol{X}$ and the ground-truth image $\boldsymbol{Y}$ both of size $(\boldsymbol{M} \times \boldsymbol{N})$ pixel is given in Eq.10, where a lower NAE points to a higher similarity.

$$
N A E=\sum_{m=1}^{M} \sum_{n=1}^{N}|(X(m, n)-Y(m, n))| / \sum_{m=1}^{M} \sum_{n=1}^{N}|X(m, n)|
$$

7. The Global Consistency Error (GCE) is a region-based segmentation consistency metric, which is computed to quantify the consistency between two segmentations and to what extent that the segmented image can be viewed as a refinement of the ground-truth image. The GCE between segmented image $\boldsymbol{X}$ and the ground-truth image $\boldsymbol{Y}$ is given by Eq.9, and ranges between $\mathbf{0}$ and $\mathbf{1}$, a lower value indicates greater similarity.

$$
\operatorname{GCE}(X, Y)=\frac{1}{n} \min \left\{\sum_{i} E\left(X, Y, p_{i}\right), \sum_{i} E\left(Y, X, p_{i}\right)\right\}
$$




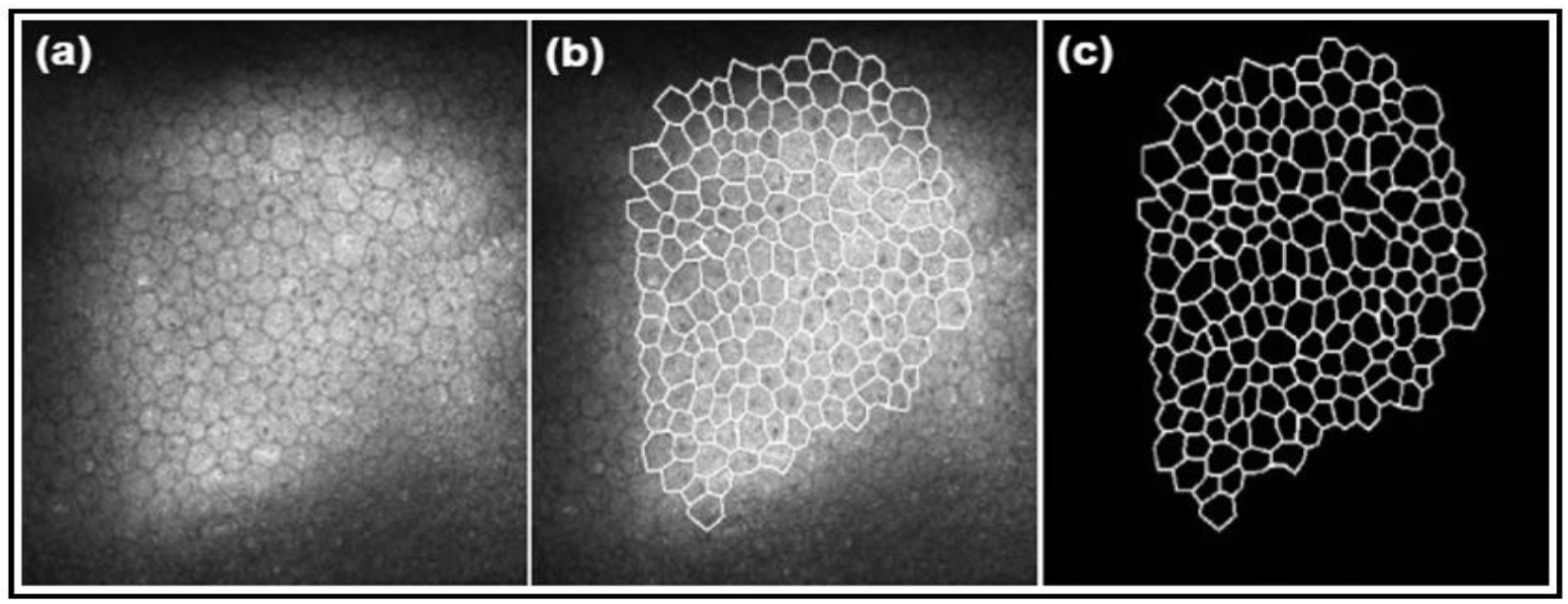

Figure 8. GIMP program outputs: (a) Original image, (b) A representative example of manually traced cell contours, (c) Generated binary image used as a ground-truth manual segmentation inside the ROI.

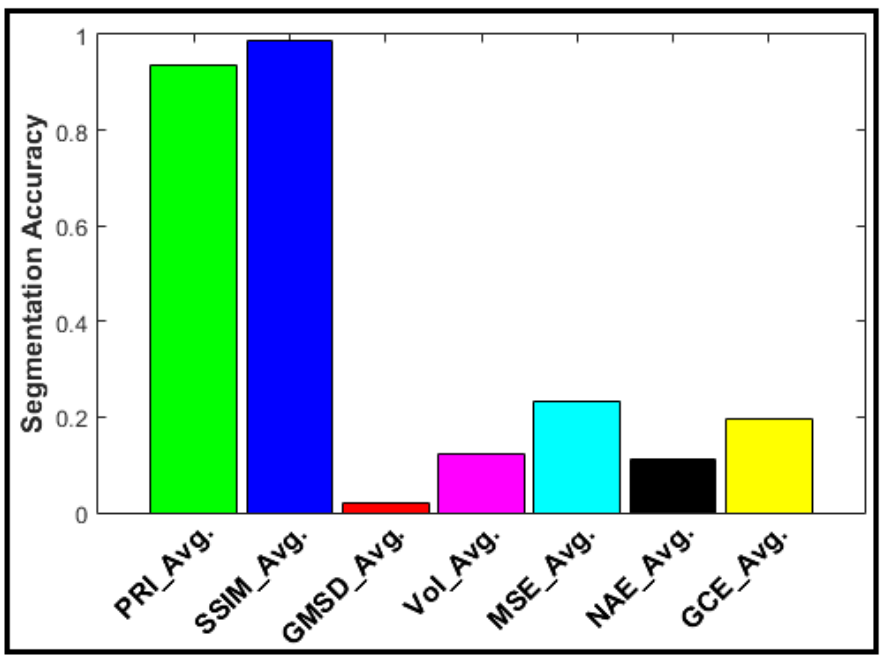

Figure 9. Descriptive statistics of the CEAS segmentation system performance on Database_1, where a higher value of PRI and SSIM is better and a lower value of GMSD, VoI MSE, NAE and GCE is better.

Here, $\boldsymbol{E}\left(\boldsymbol{X}, \boldsymbol{Y}, \boldsymbol{p}_{\boldsymbol{i}}\right)$ represents the local refinement error between segmented image $\boldsymbol{X}$ and the ground-truth image $\boldsymbol{Y}$.

The overall average of the seven quantitative metrics is computed for 40 images in this database, as shown in Fig.9. Significantly, the results obtained demonstrate the efficiency and reliability of the proposed CEAS system, and the potentiality of using it as a fully-automatic system to accurately trace cell contours and measure the morphometric parameters for clinical diagnostic purposes, due to the high similarity rates obtained between the automatically segmented images and the manually traced images. Furthermore, a simple experiment was conducted to testing the segmentation accuracy between the automatically segmented output using the proposed CEAS system and the ground-truth manual segmentation (Fig. $8 \mathrm{c}$ ). In this experiment, the segmentation overlap between the manual and automatic outputs was done using the BestFit system ${ }^{3}$ [39], and then the similarity of the cell border location for both images is produced, as descried in [40] and shown in Fig. 10. The red line describes the ground-truth manual image, blue the line the output of the CEAS system, while the black line shows the common border.

Here, it is important to note that most of the segmentation approaches and commercially available image software systems (e.g., GIMP) are edge-based segmentation approaches, whereas

\footnotetext{
${ }^{3}$ home.agh.edu.pl/ pioro/bestfit
}

in this study an alternative region-based segmentation approach based on the Voronoi Tessellation is employed to accurately extract size and shape data of the endothelial cells. In this work, using the Voronoi Tessellation, as a region-based segmentation approach ensures that a wider range of corneal endothelial parameters can be derived and analysed than edge-based segmentation approaches, by dividing the surface of the endothelial image into different regions (Voronoi cells) based on the distance to the cell markers' centres. In addition, the Voronoi Tessellation approach produces straight-borders of cells of optimum size and shape compared to the edge-based segmentation approaches, which produce cell shapes composed of non-uniform curves. This will enhance the reliability of the proposed CEAS system in calculating polymegathism and pleomorphism data of the endothelial cells [41]. Finally, as reported by Reem [41], the Voronoi Tessellation approach provides a high degree of geometric stability with respect to small changes in the position of the cell markers' centres, with only a small change in the corresponding Voronoi cells.

In the second experiment, a clinical evaluation procedure was performed in order to accurately assess the robustness and effectiveness of the CEAS system in term of extracting useful morphometric parameters. In the work, automatic estimations of five morphometric parameters (e.g., MCD, MCA, MCP, Polymegathism, and Pleomorphism) were compared with reference values, which were calculated by simply applying the definition of these parameters on 40 binary images generated using GIMP software (Fig.8 c). Firstly, the output from the proposed CEAS system was generated from each corneal endothelial image, and then the same ROI was captured with the largest area of clearly visible cells and selected to be used as a representative image for that input image. Next, an automatic estimation of morphometric parameters was computed by the proposed CEAS system for this ROI and directly compared with the reference values. The overall average, standard deviation, maximum and minimum of each parameter for both manual and automatic images, along with the difference and the percentage difference between them are reported in Table 1. No significant difference was found between manual and automatic estimations of morphometric parameters. It is interesting to note that the average difference between manual and automatic estimations was less than $2 \%, 3.5 \%, 1 \%, 1.5 \%$, and $8.5 \%$ for MCD, MCA, MCP, Polymegathism, and Pleomorphism, respectively. Generally, no morphometric parameter has a relative difference (> 10\%) between the manual and automatic estimations. Pearson correlation tests were used to confirm clinical validity and usefulness of the proposed CEAS system as an effective tool to 


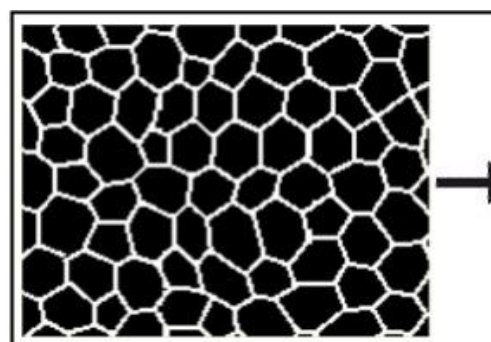

(a) Manual Image

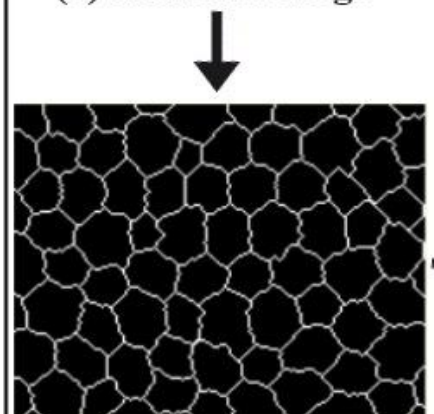

(e) Manual after BestFit

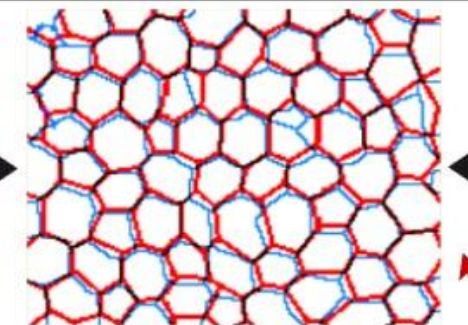

(b) Manual \& CEAS Difference

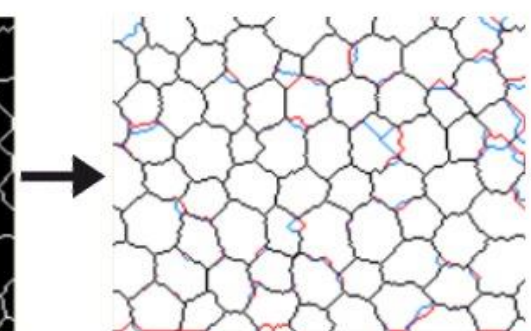

(f) Manual \& CEAS after BestFit - Difference

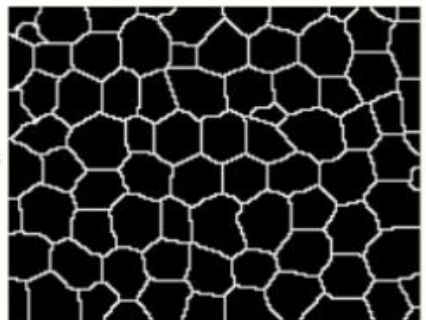

(c) Output of the CEAS

1

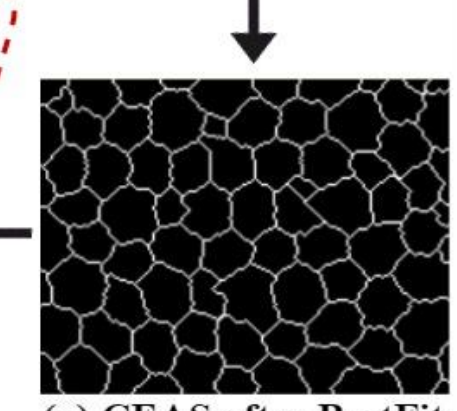

(g) CEAS after BestFit

Figure 10. Segmentation differences between the manual and automatic output obtained using CEAS system, where red and blue lines in (b) describe the ground-truth manual image and the output of the CEAS system, respectively. While the black line shows the common border between them.

Table 1. Performance comparison made between the manual and automated estimations of five morphometric parameters in 40 corneal images of Database_1. The differences between the manual and automatic estimates are listed and also as a percentage.

\begin{tabular}{|c|c|c|c|c|}
\hline & Manual MCD (cells $/ \mathrm{mm}^{2}$ ) & Auto. MCD (cells $/ \mathrm{mm}^{2}$ ) & Diff & Diff \% \\
\hline Average & 3103.52 & 3047.25 & 56.27 & 1.83 \\
\hline STD & 389.54 & 308 & 81.54 & 23.35 \\
\hline Max & 3760 & 3640 & 120 & 3.24 \\
\hline \multirow[t]{2}{*}{ Min } & 2243 & 2482 & -239 & -10.1 \\
\hline & Manual MCA $\left(\mu \mathrm{m}^{2}\right)$ & Auto. MCA $\left(\mu \mathrm{m}^{2}\right)$ & Diff & Diff \% \\
\hline Average & 292.3 & 282.43 & 9.87 & 3.43 \\
\hline STD & 41.77 & 34.57 & 7.2 & 18.87 \\
\hline Max & 395 & 386 & 9 & 2.3 \\
\hline \multirow[t]{2}{*}{ Min } & 229 & 220 & 9 & 4 \\
\hline & Manual MCP $(\mu \mathrm{m})$ & Auto. MCP $(\mu \mathrm{m})$ & Diff & Diff \% \\
\hline Average & 61.5 & 61 & 0.5 & 0.78 \\
\hline STD & 4.61 & 3.52 & 1.09 & 26.8 \\
\hline Max & 74 & 70 & 4 & 5.56 \\
\hline \multirow[t]{2}{*}{ Min } & 55 & 54 & 1 & 1.83 \\
\hline & Manual Polymegathism \% & Auto. Polymegathism \% & Diff & Diff \% \\
\hline Average & 46.5 & 45.95 & 0.55 & 1.02 \\
\hline STD & 5.96 & 4.68 & 1.28 & 24.09 \\
\hline Max & 61 & 59 & 2 & 3.33 \\
\hline \multirow[t]{2}{*}{ Min } & 36 & 37 & -1 & -2.74 \\
\hline & Manual Pleomorphism \% & Auto. Pleomorphism \% & Diff & Diff \% \\
\hline Average & 37.45 & 34.5 & 2.95 & 8.21 \\
\hline STD & 5.84 & 4.83 & 1.01 & 18.84 \\
\hline Max & 50 & 47 & 3 & 6.2 \\
\hline Min & 24 & 25 & -1 & -4.1 \\
\hline
\end{tabular}



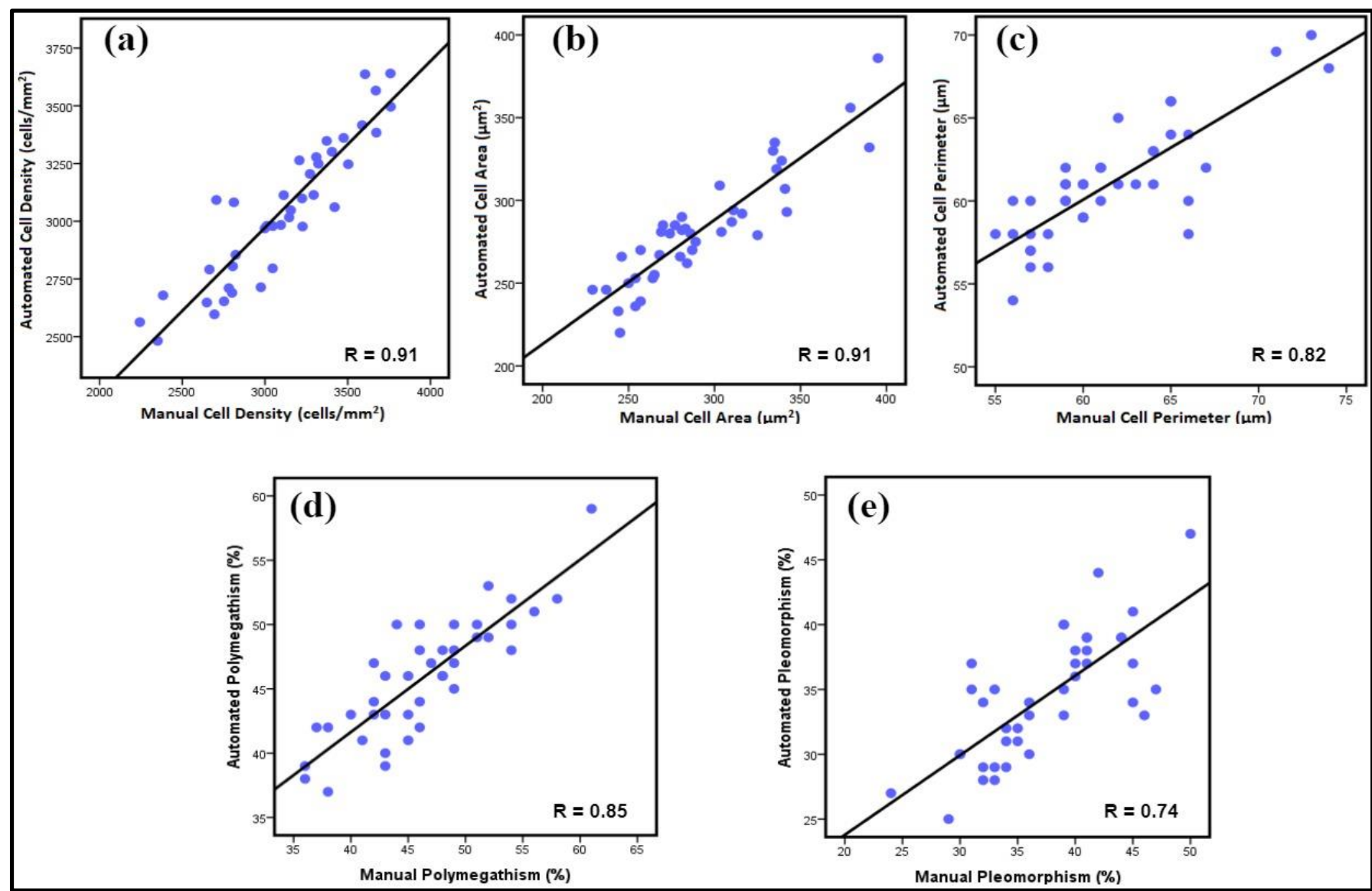

Figure 11. Correlation plots for each pair of manual and automatic morphometric parameters from Database_l, showing significant correlations. The solid lines are the linear regression lines: (a) Cell density, (b) Cell area, (c) Cell perimeter, (d) Polymegathism, and (e) Pleomorphism.

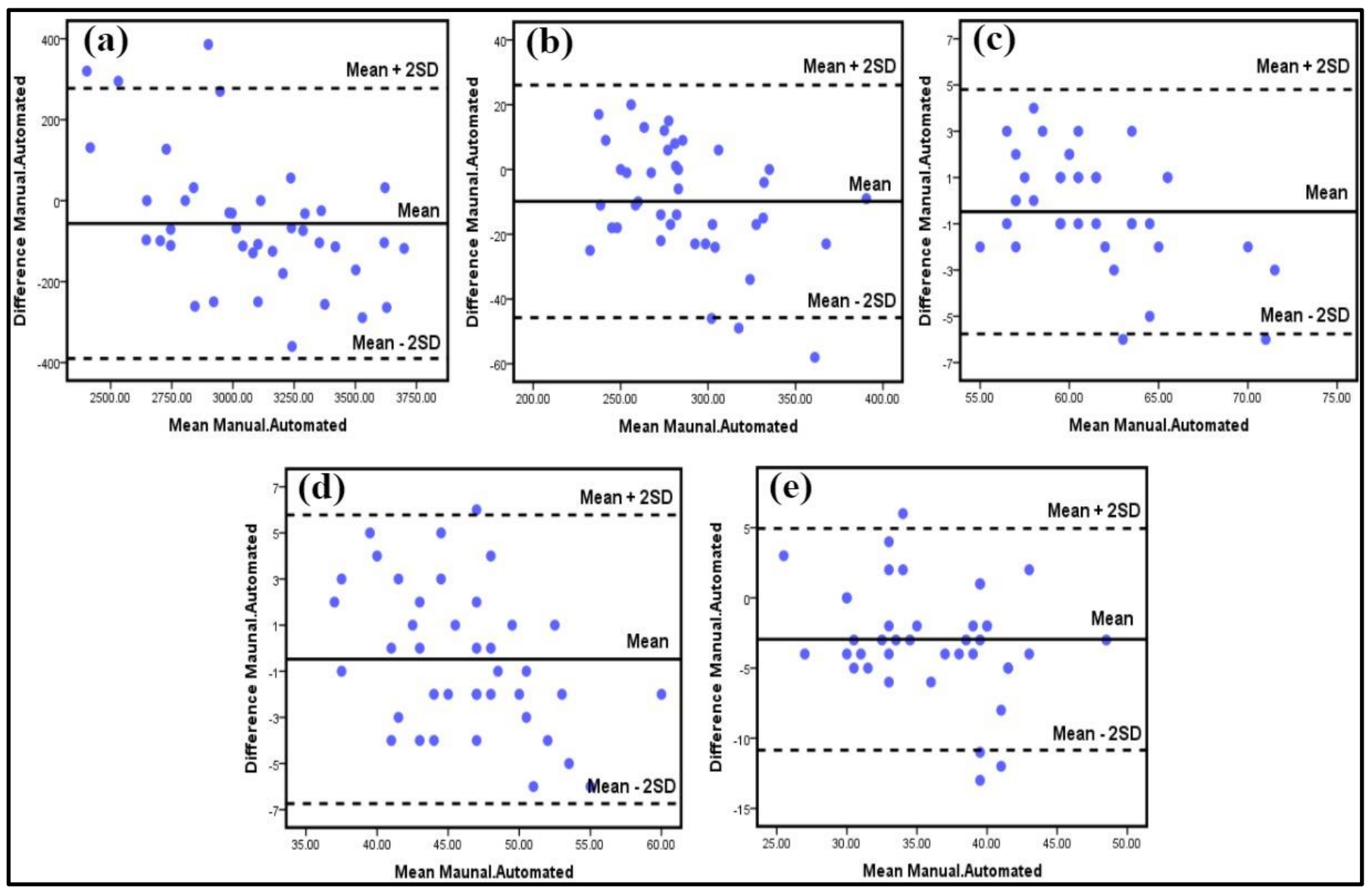

Figure 12. Bland-Altman plots showing difference versus average for each pair of manual and automatic estimations of: (a) Cell density, (b) Cell area, (c) Cell perimeter, (d) Polymegathism, and (e) Pleomorphism from Database_1. Solid lines (mean differences), dashed lines (95\% limits of agreement). 
Table 2. Performance comparison between the manual and automated cell density estimates on the Database_2

\begin{tabular}{ccccc}
\hline & Manual MCD (cells $\left./ \mathrm{mm}^{2}\right)$ & Auto. MCD $\left(\right.$ cells $\left./ \mathrm{mm}^{2}\right)$ & Diff & Diff $\%$ \\
\hline Average & 3390.18 & 3343.65 & 46.53 & 1.38 \\
STD & 715 & 415 & 300 & 53.01 \\
Max & 4960 & 4247 & 713 & 15.49 \\
Min & 1941 & 2473 & -532 & -24.1 \\
\hline
\end{tabular}

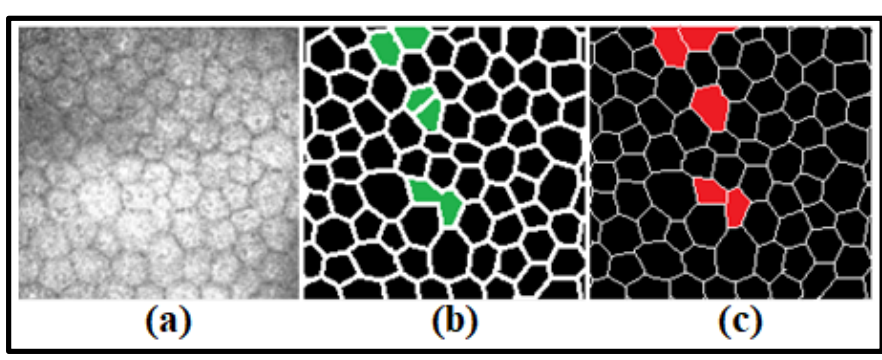

Figure 13. Comparison between manual and automated segmentation outputs: (a) Original image, (b) Manually traced cells, (c) Automatically segmented cells.

provide an accurate and automatic estimation of the endothelial cell parameters. There were statistically significant correlations between the automated and manual estimations of morphometric parameters with Pearson's correlation $r$ and $p$ coefficient of: $(r=$ $0.91, \mathrm{p}<0.0001)$ for MCD, $(r=0.91, \mathrm{p}<0.0001)$ for MCA, $(\mathrm{r}=$ $0.82, \mathrm{p}<0.0001)$ for MCP, $(\mathrm{r}=0.85, \mathrm{p}<0.0001)$ for polymegathism, and $(\mathrm{r}=0.74, \mathrm{p}<0.0001)$ for pleomorphism (Fig.11). Bland-Altman plots of differences versus means for all the morphometric parameters were generated to assess agreement between the automated analysis and manual analysis (Fig.12).The proposed CEAS system produces an accurate estimation for detecting endothelial cells with more than $95 \%$ of the data presented between 2SD agreement lines, and cell densities ranging from 2400 to $3700\left(\right.$ cell $\left./ \mathrm{mm}^{2}\right)$, cell area ranging from 230 to $400\left(\mu \mathrm{m}^{2}\right)$, cell perimeter ranging from 50 to 75 $(\mu \mathrm{m})$, polymegathism ranging from 35 to $40 \%$, and pleomorphism ranging from 25 to $50 \%$. Fig. 13 shows a comparison between manual and automated segmentation output images, where the incorrectly detected cell boundaries are marked in red color.

Further evaluation was performed on an independent database, named Database_2 in which a manual database containing 40 images (11 from control subjects, 16 from obese subjects and 13 from patients with diabetes) was constructed to efficiently validate the performance of the proposed corneal cell segmentation and quantification system. The cell densities were manually counted by an expert from the University of Manchester using a semi-automatic system (cell count feature) offered by the HRT Rostock Cornea Module (Heidelberg Engineering $\mathrm{GmbH}$; Heidelberg; Germany). In the manual measurements, the user selects the clearest ROI from the original corneal endothelial image and then crops it. After magnifying the cropped region to make it easier to view, the user picks the cells using the count function and the MCD is calculated according to the number of cells within the given ROI. A snapshot of this semi-automatic system is shown in Fig.14. These manual cell densities were compared with automatically computed cell densities using the CEAS system for the same ROI. The results demonstrate the ability of the proposed CEAS system to detect corneal endothelial cells effectively in clinical real-time, with an execution time of about 6 seconds per image using a PC with a Windows 8.1 operating system, a $1.80 \mathrm{GHz}$ Core i5-3337U CPU and 6 GB of RAM. The system code was written in MATLAB $\mathrm{R} 2015 \mathrm{a}$. The main reason for differences between the manual and automated cell densities is the loss of image quality at the borders of the images, and there may be some cells in the cropped ROI, which are over selected in the manual image or are not picked at all (Fig.15). As shown in Table 2, there was no significant difference in manual compared to automated endothelial cell density and the average difference was less than $2 \%$. There was a highly significant correlation between automated and manual densities $(r=0.9, \mathrm{p}<0.0001)$ (Fig.16). The proposed segmented endothelial cell detection algorithm provides a precise estimation for detecting endothelial cells with cell densities ranging from 2000 to 5000 (cells $/ \mathrm{mm}^{2}$ ) and the Bland-Altman method shows that $95 \%$ of data are presented between 2SD agreement lines. Based on the agreement plot the difference between the two methods is larger when the density is higher (Fig.17). The results obtained demonstrate the effectiveness and robustness of the proposed CEAS system, and its suitability to be used as a fully automatic cell segmentation system to provide useful clinical information for early diagnosis and monitoring of the corneal endothelium over time and in relation to the effect of therapies, by achieving a high similarity between the cell density obtained automatically and the cell density obtained manually.

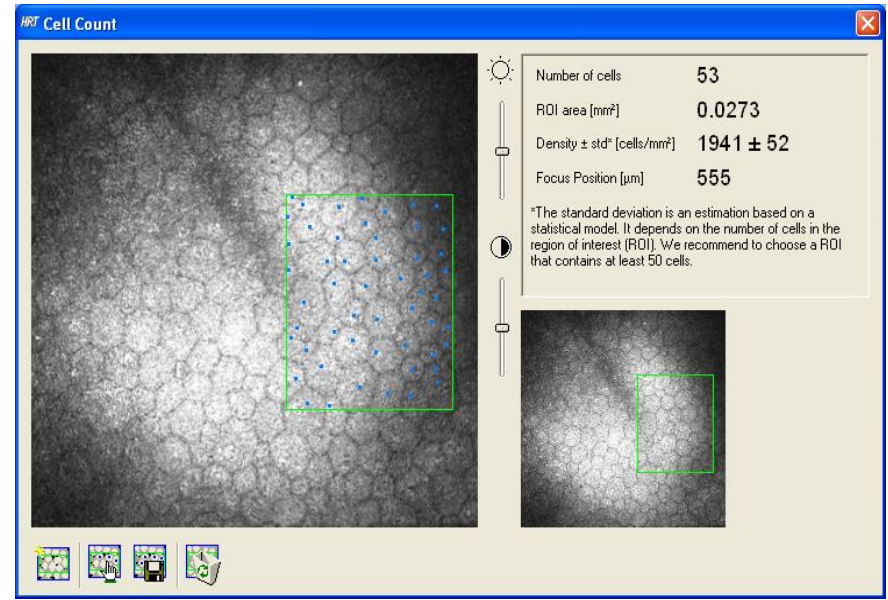

Figure 14. An illustration of the semi-automatic corneal endothelium system for cell density estimation.

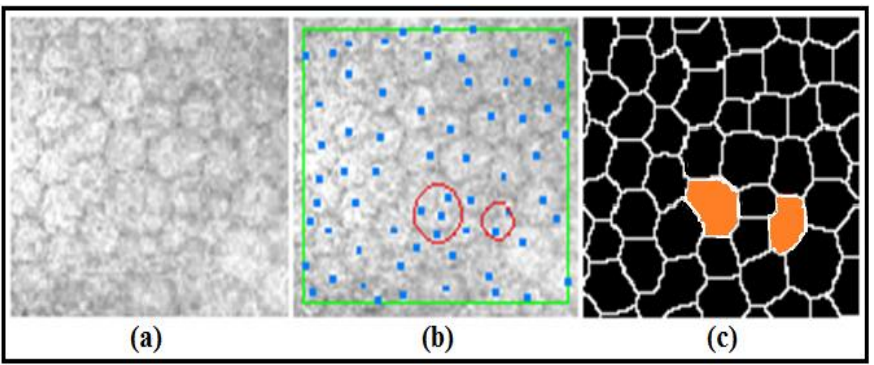

Figure 15. (a) the original image, (b) Manually over picked cells as shown in the red circle, (c) Automatically segmented cells. 


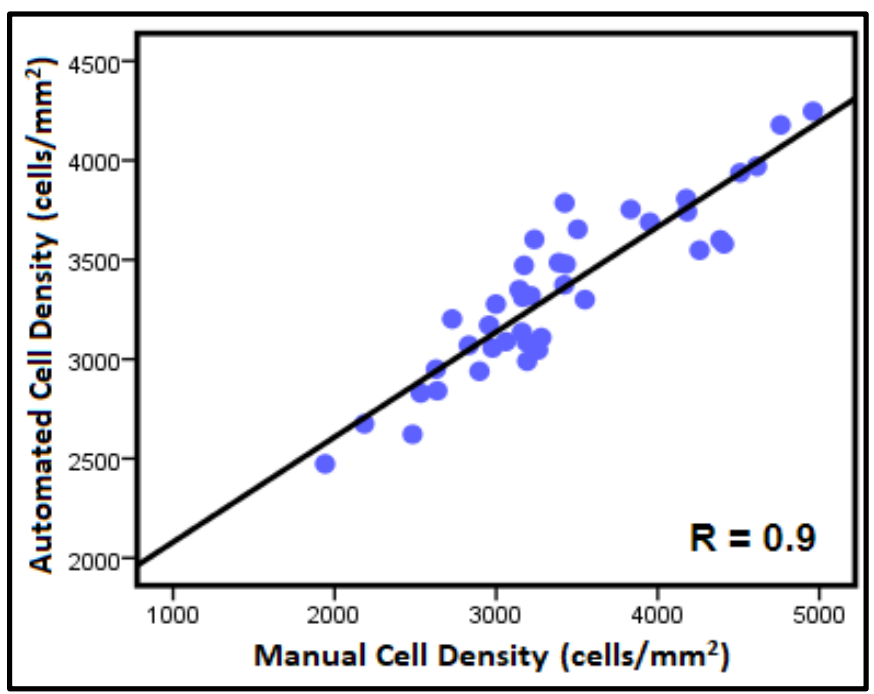

Figure 16. Correlation plot of automated and manual cell densities with a significant correlation on Database_2

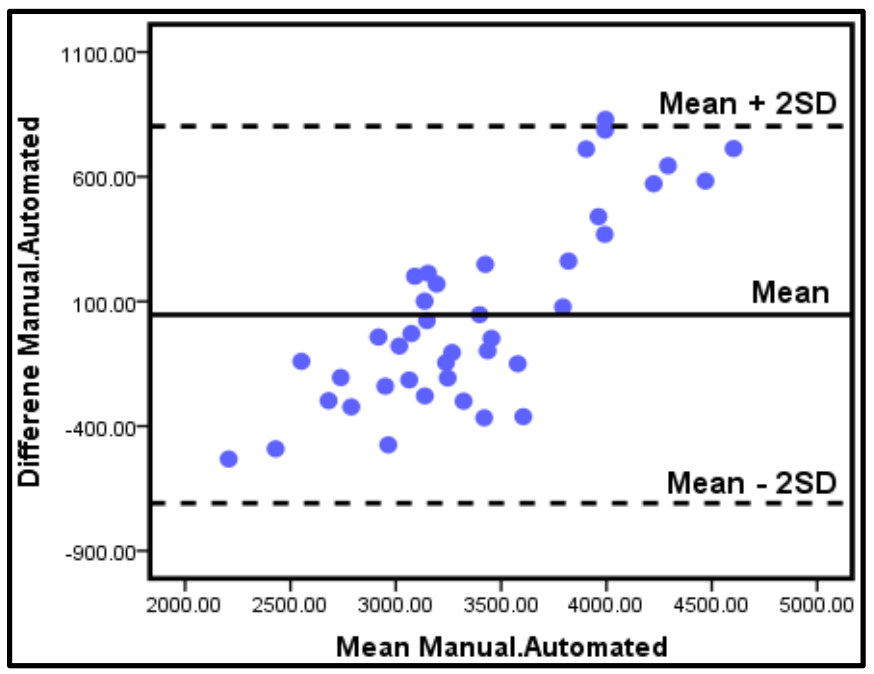

Figure 17. Bland-Altman plot showing mean difference and limits of agreement between manual and automated cell densities on Database_2.

\section{Conclusions and Future Work}

In this paper, a real-time and fully automated endothelial cell segmentation and morphological parameter quantification system is proposed, named the Corneal Endothelium Analysis System (CEAS) which requires no user intervention. In the CEAS system, A FFT-Band-pass filter was applied for noise reduction to enhance the image quality and endothelial cell boundaries were detected using the watershed approach and Voronoi tessellations enabling quantification of endothelial cell density and additionally cell area, cell perimeter, polymegathism and pleomorphism. The extracted clinical features from two databases (each one consisting of 40 images), were compared with 'ground-truth' derived by manually detecting the cell contours aided by two difference programs on the same ROI with an average difference of less than $2 \%, 4 \%, 1 \%, 1.5 \%, 8 \%$ for MCD, MCA, MCP, polymegathism, and pleomorphism, respectively. This shows the efficiency and reliability of the CEAS system, and the possibility of utilizing it in a real world clinical setting to enable rapid diagnosis and patient follow-up. This promising pilot data encourages us to develop an automated machine learning system for the early diagnosis of endothelial cell abnormalities. Hence, we need a larger image database to construct a learning-based module, and feed it with the 5 morphometric parameters extracted from each subject to produce a more comprehensive morphological feature vector for each subject, to enable more reliable diagnosis and confirm our findings.

\section{Conflicts of Interest}

The authors declare no conflict of interest.

\section{References}

[1] T. Zheng, Q. Le, J. Hong, and J. Xu, "Comparison of human corneal cell density by age and corneal location: an in vivo confocal microscopy study," BMC Ophthalmol., vol. 16, no. 1, p. 109, 2016.

[2] S. Al-Fahdawi, R. Qahwaji, A. S. Al-Waisy, and S. Ipsopn, "An automatic corneal subbasal nerve registration system using FFT and phase correlation techniques for an accurate DPN diagnosis," Comput. Inf. Technol. Ubiquitous Comput. Commun. Dependable, Auton. Secur. Comput. Pervasive Intell. Comput. (CIT/IUCC/DASC/PICOM), 2015 IEEE Int. Conf. on. IEEE, pp. 1035-1041, 2015

[3] M. S. Sharif, R. Qahwaji, E. Shahamatnia, R. Alzubaidi, S. Ipson, and A. Brahma, "An efficient intelligent analysis system for confocal corneal endothelium images," Comput. Methods Programs Biomed., vol. 122, no. 3, pp. 421-436, 2015.

[4] B. Selig, K. A. Vermeer, B. Rieger, T. Hillenaar, and C. L. Luengo Hendriks, "Fully automatic evaluation of the corneal endothelium from in vivo confocal microscopy.," BMC Med. Imaging, vol. 15, no. 1, p. 13,2015

[5] M. Foracchia and A. Ruggeri, "Corneal endothelium cell field analysis by means of interacting bayesian shape models," Annu. Int. Conf. IEEE Eng. Med. Biol. - Proc., pp. 6035-6038, 2007.

[6] F. Scarpa and A. Ruggeri, "Segmentation of Corneal Endothelial Cells Contour by Means of a Genetic Algorithm," in Proceedings of the Ophthalmic Medical Image Analysis Second International Workshop, OMIA 2015, Held in Conjunction with MICCAI 2015, Munich, Germany, 2015, pp. 25-32.

[7] E. Hatipoglu, C. Arici, O. S. Arslan, F. Dikkaya, P. Sultan, P. Kadioglu, and S. Gundogdu, "Corneal endothelial cell density and morphology in patients with acromegaly," Growth Horm IGF Res, vol. 24 , no. 6, pp. 260-263, 2014

[8] J. Navaratnam, T. P. Utheim, V. K. Rajasekhar, and A. Shahdadfar, "Substrates for Expansion of Corneal Endothelial Cells towards Bioengineering of Human Corneal Endothelium.," J. Funct. Biomater., vol. 6, no. 3, pp. 917-45, 2015.

[9] B. E. McCarey, H. F. Edelhauser, and and M. J. Lynn, "Review of Corneal Endothelial Specular Microscopy for FDA Clinical Trials of Refractive Procedures, Surgical Devices and New Intraocular Drugs and Solutions," Cornea, vol. 27, no. 1, pp. 1-16, 2008.

[10] A. Ruggeri, E. Grisan, and J. Jaroszewski, "A new system for the automatic estimation of endothelial cell density in donor corneas," $\mathrm{Br}$. J. Ophthalmol., vol. 89, no. 3, pp. 306-11, 2005.

[11] P. Gain, G. Thuret, L. Kodjikian, Y. Gavet, P. H. Turc, C. Theillere, S Acquart, J. C. Le Petit, J. Maugery, and L. Campos, "Automated triimage analysis of stored corneal endothelium," Br. J. Ophthalmol., vol. 86 , no. 7, pp. 801-8, 2002

[12] M. J. Doughty and B. M. Aakre, "Further analysis of assessments of the coefficient of variation of corneal endothelial cell areas from specular microscopic images," Clin. Exp. Optom., vol. 91, no. 5, pp. 438-446, 2008.

[13] M. Foracchia and A. Ruggeri, "Cell contour detection in corneal endothelium in-vivo microscopy," Proc. 22nd Annu. Int. Conf. IEEE Eng. Med. Biol. Soc., vol. 2, pp. 1033-1035, 2000.

[14] E. Grisan, A. Paviotti, N. Laurenti, and A. Ruggeri, "A lattice estimation approach for the automatic evaluation of corneal endothelium density," Conf. Proc. ... Annu. Int. Conf. IEEE Eng. Med. Biol. Soc. IEEE Eng. Med. Biol. Soc. Annu. Conf., vol. 2, no. 1, pp. 1700-3, 2005.

[15] T. Hiroyasu, S. Sekiya, S. Nunokawa, N. Koizumi, N. Okumura, and U. Yamamoto, "Extracting rules for cell segmentation in corneal endothelial cell images using GP," Proc. - 2013 IEEE Int. Conf. Syst. Man, Cybern. SMC 2013, pp. 1811-1816, 2013.

[16] E. Poletti and A. Ruggeri, "Segmentation of Corneal Endothelial Cells Contour through Classification of Individual Component Signatures," IFMBE Proc., vol. 41, pp. 658-661, 2014. 
[17] S. Katafuchi and M. Yoshimura, "Convolution neural network for contour extraction of corneal endothelial cells," Katafuchi, S., Yoshimura, M. (2017, May). Convolution neural Netw. contour Extr. corneal Endothel. cells. Thirteen. Int. Conf. Qual. Control by Artif. Vis. 2017, vol. 10338, p. 103380L, 2017.

[18] A. Fabijańska, "Corneal Endothelium Image Segmentation Using Feedforward Neural Network," in Proceedings of the Federated Conference on Computer Science and Information Systems, 2017, vol. 11, pp. 629-637.

[19] Y. Gavet and J. C. Pinoli, "Comparison and supervised learning of segmentation methods dedicated to specular microscope images of corneal endothelium," Int. J. Biomed. Imaging, vol. 2014, no. ii, 2014.

[20] K. Habrat, M. Habrat, J. Gronkow-Skaserafin, and A. Piórkowski, "Cell detection in corneal endothelial images using directional filters," Adv. Intell. Syst. Comput., vol. 389, pp. 113-123, 2015.

[21] M. Tavakoli and R. A. Malik, "Corneal Confocal Microscopy: A Novel Non-invasive Technique to Quantify Small Fibre Pathology in Peripheral Neuropathies," J. Vis. Exp., no. 47, pp. 1-7, 2011.

[22] M. S. Sharif, R. Qahwaji, S. Hayajneh, S. Ipson, R. Alzubaidi, and A. Brahma, "An efficient system for preprocessing confocal corneal images for subsequent analysis," 2014 14th UK Work. Comput. Intell. UKCI 2014 - Proc., 2014.

[23] J.W. Cooley and J.W. Tukey, "An algorithm for the machine calculation of complex Fourier Series," Math. Comput., vol. 19, no. 90 p. pp 297-301, 1965.

[24] R. Gonzalez and R. Woods, Digital image processing. Digital image processing, 2008.

[25] S. W. Smith, "Digital signal processing. " California Technical Publishing, 1999.

[26] M. N. Haque and M. S. Uddin, "Accelerating Fast Fourier Transformation for Image Processing using Graphics Processing Unit," J. Emerg. Trends Comput., vol. 2, no. 8, pp. 367-375, 2011.

[27] F. Meyer, "The watershed concept and its use in segmentation : a brief history," arXiv preprint arXiv, pp. 1-11, 2012.

[28] S. Beucher and C. Lantuejoul, "Use of Watersheds in Contour Detection," Int. Work. Image Process. Real-time Edge Motion Detect. no. JANUARY 1979, pp. 12-21, 1979.

[29] S. Beucher and F. Meyer, "The Morphological Approach to Segmentation: The Watershed Transformation," in Optical Engineering-New York-Marcel Dekker Incorporated- 34, 1993, pp. 433-481.

[30] Y.-Y. Kim, B.-M. Kim, H.-J. Park, K.-B. Im, J.-S. Lee, and D.-Y. Kim "Image Analysis Algorithm for the Corneal Endothelium," J. Biomed. Eng. Res., vol. 27, no. 3, pp. 125-130, 2006.

[31] N. H. Brookes, "Morphometry of organ cultured corneal endothelium using Voronoi segmentation," Cell Tissue Bank., vol. 18, no. 2, pp. 167-183, 2017.

[32] J. Kaur, S. Agrawal, and R. Vig, "Integration of Clustering, Optimization and Partial Differential Equation Method for Improved Image Segmentation," Int. J. Image, Graph. Signal Process., vol. 4, no. 11, pp. 26-33, 2012.

[33] Z. Wang, A. C. Bovik, H. R. Sheikh, S. Member, E. P. Simoncelli, and S. Member, "Image Quality Assessment: From Error Visibility to Structural Similarity," IEEE Trans. IMAGE Process., vol. 13, no. 4, pp. 1-14, 2004.

[34] W. Xue, L. Zhang, X. Mou, and A. C. Bovik, "Gradient magnitude similarity deviation: A highly efficient perceptual image quality index," IEEE Trans. Image Process., vol. 23, no. 2, pp. 668-695, 2014.

[35] M. Meilă, "Comparing Clusterings - An Information Based Distance," J. Multivar. Anal., vol. 98, no. 5, pp. 873-895, 2007.

[36] K. Mallikarjuna, K. Satya Prasad, and M. Venkata Subramanyam, "Image Compression and Reconstruction using Discrete Rajan Transform Based Spectral Sparsing," Int. J. Image, Graph. Signal Process., vol. 8, no. 1, pp. 59-67, 2016.

[37] D. Martin, C. Fowlkes, D. Tal, and J. Malik, "A database of human segmented natural images and its application toevaluating segmentation algorithms and measuring ecological statistics," Proc Eighth IEEE Int. Conf. Comput. Vision. ICCV 2001, vol. 2, no. July, 2001.

[38] S. Al-Fahdawi, R. Qahwaji, A. S. Al-Waisy, S. Ipson, R. A. Malik, A Brahma, and X. Chen, "A fully automatic nerve segmentation and morphometric parameter quantification system for early diagnosis of diabetic neuropathy in corneal images," Comput. Methods Programs Biomed., vol. 135, pp. 151-166, 2016.

[39] A. Piórkowski, "Best-Fit Segmentation Created Using Flood-Based Iterative Thinning," Springer, vol. 525, pp. 61-68, 2017.

[40] A. Piorkowski, K. Nurzynska, J. Gronkowska-Serafin, B. Selig, C. Boldak, and D. Reska, "Influence of applied corneal endothelium image segmentation techniques on the clinical parameters," Comput. Med. Imaging Graph., vol. 55, pp. 13-27, 2017.

[41] D. Reem, "The geometric stability of Voronoi diagrams with respect to small changes of the sites," in In Proceedings of the twenty-seventh annual symposium on Computational geometry, ACM, 2011, pp. 254263. 